American Journal of BioScience
2017;5(5): $80-88$
http://www.sciencepublishinggroup.com/j/ajbio
doi: $10.11648 /$ jajbio.20170505.12
ISSN: 2330 -0159 (Print); ISSN: $2330-0167$ (Online)

\title{
Community Knowledge, Attitude and Practice About Malaria and Mosquito Biting Behavior in Southern Ethiopia
}

\author{
Temam Abrar Hamza ${ }^{1}$, Nuredin Nassir Azmach ${ }^{2}$, Awel Abdella Husen ${ }^{3}$ \\ ${ }^{1}$ Department of Bio-technology, Arba Minch University, Arba Minch, Ethiopia \\ ${ }^{2}$ Department of Statistics, Arba Minch University, Arba Minch, Ethiopia \\ ${ }^{3}$ Department of Physics, Arba Minch University, Arba Minch, Ethiopia
}

Email address:

temam2abrar2@gmail.com (T. A. Hamza),nurnas20@gmail.com (N. N. Azmach), abdelaawel@gmail.com (A. A. Husen)

\section{To cite this article:}

Temam Abrar Hamza, Nuredin Nassir Azmach, Awel Abdella. Community Knowledge, Attitude and Practice About Malaria and Mosquito Biting Behavior in Southern Ethiopia. American Journal of BioScience. Vol. 5, No. 5, 2017, pp. 80-88. doi: 10.11648/j.ajbio.20170505.12

Received: July 9, 2017; Accepted: July 21, 2017; Published: October 26, 2017

\begin{abstract}
Malaria has been the leading cause of mortality for many years in underdeveloped countries like Ethiopia. Community Knowledge of cause and transmission of malaria and mosquito feeding behavior is key to design appropriate health communication strategies for malaria prevention and control. The present study aimed to assess knowledge and awareness on malaria and its association with mosquitoes in Dembele kebele, southeastern, Ethiopia. A community cross sectional study was carried out in Dembele kebele from January to February, 2017. A total of 159 randomly selected individuals were interviewed to assess their KAP about malaria. Data were collected by an interview based structured questionnaire after pre-tested. The collected data were entered in to SPSS for data coding and analysis. Binary logistic regressions analysis was applied and statistical significance test was declared at P-value $<0.05$ and OR with $95 \%$ CI. All respondents had ever heard of malaria and more than $86 \%$ of them believed that malaria was one of the most health problem diseases of the community. Most of the respondents (70.4\%) were reported mosquito bite as the cause of malaria. The majority (79.2\%) and (74.2\%) of respondents had good levels of knowledge on transmission and prevention of malaria respectively. Binary logistic regression analysis showed that, knowledge on mode of malaria transmission was positively associated with sex (male) $(\mathrm{OR}=3.3$; $\mathrm{CI}, 1.27-7.22)$, age $(\mathrm{OR}=5.03$; $\mathrm{CI}, 1.03-24.46)$ and education level $(\mathrm{OR}=3.30$; 95\% CI, 1.04-10.50). Among socio-demographic variables, only education level $(\mathrm{OR}=3.8 ; \mathrm{CI}, 1.51-9.65)$ was significance factor to knowledge on malaria prevention. The study aimed to assess knowledge and awareness regarding the cause, transmission and preventive measures of malaria; it was found to be high. Use of mosquito net as protective measure against mosquito bites was high in the study area. However, distance of respondent house is near to the mosquito breeding site. Therefore, environmental management and use of other additional preventive methods is very important to reduce prevalence of malaria in study area. Sex, age and education level were strong predictors of knowledge on malaria.
\end{abstract}

Keywords: Malaria, Mosquito, Knowledge, Attitude, Preventive, Control, Dembele, Ethiopia

\section{Introduction}

Malaria is one of the major public health challenges undermining development in underdeveloped countries like Ethiopia. It remains a threat to almost $50 \%$ of the world's population with 300-500 million annual clinical cases and kills up to 3 million people annually of whom $90 \%$ of the cases and deaths occur in Africa [1,2]. It is an infectious disease and a result of protozoan parasite from the genus plasmodium which is transmitted by female anopheles mosquito in turn infects people [3, 4, 5]. Four species of plasmodium infects human beings, these are Plasmodium vivax, Plasmodium falciparum, Plasmodium ovalae and plasmodium malarae [1]. Among the four plasmodium species known to cause malaria in Ethiopia, the two epidemiologically important species are Plasmodium falciparum and Plasmodium vivax comprising $60 \%$ and $40 \%$ respectively [6].

Malaria transmission was remarkably associated with rural environments due to mosquito's affinity for clean, and temporally pools of water, which are the most important in 
rural areas as anopheline larval sites [7, 8]. Risk factors for malaria infection include location (house near to breeding site and larval habitats) [1], attributes of houses or living units [9], socio-economic factors [1, 10] and genetic characteristics of the occupants [11]. The climatic change like heavy rain and higher temperature favor the breeding of mosquitoes. The study conducted in Eddo Kontola was indicated that, people living nearest of Lake Zway had more affected with malaria than those living far from the lake [12]. In rural setting, incidence of clinical episodes of malaria was strongly associated with proximity of residence to potential mosquito breeding sites $[11,13]$.

The prevalence of malaria in Dares Salaam, Tanzania among school children living in the urban area was $2-10 \%$ with predominant malaria parasite of Plasmodium falciparum accounting for $90 \%$ of all cases [7]. Reviewing evidence from Kinshasa, the capital of the Democratic Republic of the Congo, showed that the prevalence rate varied from $4 \%$ in the urban zone to $46 \%$ in the per-urban zone with plasmodium falciparum was accounted for more than $97 \%$ of the infection [1]. The prevalence of malaria in Ethiopia is highly depending on the geographical location and altitude of particular place. For instance, the prevalence of malaria in eddo Kontola, Central Ethiopia, was ranged from 3.5\% to $12.6 \%$ (average, 6.8\%) [12]; in Tigray, Northern Ethiopia, parasite rate in cross sectional surveys was ranged from 3$10 \%$ during high transmission months and from $0-3 \%$ during low transmission months [9]; in Nazareth town revealed $2.8 \%$ [14]; in Akaki town 3.7\% [15]; and a retrospective medical analysis in Gondar College of Medical Sciences indicated that, out of 6100 patients admitted, 427 (7\%) had a primary diagnosis of malaria $[16,17]$.

Generally in Ethiopia, malaria is prevalent in $75 \%$ of the country's landmass where more than 48 million people reside and annually 100,000 lives claimed. In a non-epidemic year, 5-6 million clinical malaria cases and over 600,000 confirmed cases were reported from health facilities. However, the number of malaria cases reported by health facilities was only a portion of the actual magnitude [16, 18]. According to Federal Minister of Health Office (FMHO) report, Amhara Regional state is the second highly affected next to Oromia, with malaria burden compared to the rest of largest Regions, Southern and Tigray [7, 17]. In addition, other studies shown that malaria is one of the top ten causes of illnesses and deaths in this regions [19].

The main components of malaria control strategies in Ethiopia include the early diagnosis and effective treatment of cases, the application of selective vector control measures such as indoor residual insecticide spray and environmental management [20]. However, the prevalence of malaria in the country is still high. Inadequate knowledge of local malaria vectors, their ecology and habit have contributed to challenges of the mosquito control programs [12]. On top of these, lack of public awareness on the cause, transmission and prevention methods and attitude and practice toward the use of Insecticide Treated Nets (ITNs) have also contributed to challenges of malaria prevention and control strategy in the country.
Demographic and Health Survey in Ethiopia indicated that only $1 \%$ of household has bed nets, with urban households to possess bed nets more than rural, $3 \%$ and $1 \%$ respectively.

In fact, in Ethiopia the most frequently used malaria preventive measures are draining stagnant water, sleeping under mosquito nets and indoor residual spraying [21]. Promoting active community involvement and ownership in malaria control and management through strengthening community based organizations and appropriate health education would be relevant to reduce the prevalence of malaria [22, 23, 24, 25]. Environmental management and ITNs for prevention of malaria are safe, socially acceptable and should be promoted for vector control to reduce the disease burden in the rural communities [26, 27].

Despite the effect and consequence of malaria in Ethiopia, communities based studies were not conducted at Bonke woreda, Dembele kebele. Malaria transmission was remarkably associated with rural environments due to several factories as mentioned above. Therefore, the research project was initiated to assess and explore public awareness and community attitude towards the cause, transmission and prevention of malaria.

\section{Methods}

\subsection{Study Design and Setting}

A community-based cross-sectional study was conducted in Dembele Kebele from January to February 2017, which is located in Bonke woreda, Gamo Gofa Zone. The woreda is located at $75 \mathrm{~km}$ from Arba Minch Town, city of the Zone, in the Southern Regional State, at an elevation of about 500 to 3200 meters above sea level and land area covers about 1547 hector. The average annual temperature of the woreda is $28^{\circ} \mathrm{C}$ and the average annual rain fall varying from 900 to $1900 \mathrm{~mm}$ per year, the climatic condition is belongs to temperate (woyna dega) and the distribution of rain fall examined from March to April season.

The total population of the kebele was estimated to be 6,823 from which 3535 are female and 3288 are male and 730 households in the kebele, with the average of 9 family sizes. Malaria is one of the top ten diseases in the kebele and reported throughout the year [28]. The major livelihood of the Dembele kebele is local agriculture practice and livestock production. Livestock are the assets of household, and plays an important role in people's economy in the kebele.

\subsection{Source and Study Population}

The source populations were all adults whose age $\geq 15$ years and live in Dembele kebele. Study populations were all adults whose age $\geq 15$ years systematically selected in Dembele kebele.

\subsection{Sample Size Estimation and Sampling Technique}

The sample size for the study was calculated using the formula $n=\left[\left(Z_{\frac{\alpha}{2}}{ }^{2}\right) P(1-P)\right] / D^{2}$ for estimating a single 
population proportion at $95 \%$ confidence interval (CI) $\left(Z_{\frac{\alpha}{2}}^{2}=\right.$ 1.96), $5 \%$ margin of error and $15 \%$ non-response rates. The prevalence of plasmodial infection among febrile individuals visiting a health center in the study area is $10 \%$ [28]. Calculations were based on $P=0.1$, and $n=\frac{\left[(1.96)^{2}(0.1)(0.9)\right]}{(0.05)^{2}}=139$; contingency for non response rate of $15 \%$, a maximum of 159 study subjects were required. As to the sampling procedures, an average family size of kebele is 9, so assuming an average of three individuals older than 15 years from each household a total of 159 individuals were selected from 53 households using systematic random sampling method. The overall households (730) of the kebele were divided by the required numbers of households (53) to determine a sampling interval for selecting households. Accordingly, the first household was selected by using lottery method and then every $13^{\text {th }}$ households was selected randomly through appointments.

\subsection{Data Collection Procedure and Tools}

The study instrument was an interviewer administered questionnaire was designed which comprised of four parts. Part A related to socio-demographic background, Part B on knowledge and attitude regarding malaria transmission and preventive methods, Part $\mathrm{C}$ on knowledge and attitude related to cause of malaria and mosquito breeding site and biting behavior, and Part D on housing condition and practice related to malaria prevention and control methods. The questionnaire was adapted from different literature that was used for data collection purpose. Six nurses were selected to collect data and two supervisors who are health professionals were participated. The investigators offered two day training for data collectors and supervisors. Training sessions was contains on how to conduct the interview, content of the questionnaire, data quality, ways to approach respondents, and to keep confidentiality.

\subsection{Measurements}

For knowledge regarding malaria transmission, each right response was given a score of 1 while a wrong or unsure response was scored 0 . Total knowledge scores can range between $0-5$. If the respondent answered all questions correctly were considered as having "good knowledge" otherwise considered as "poor knowledge" regarding malaria transmission. Knowledge toward malaria prevention methods was assessed using 8 items questionnaire where knowledge score between 0-5 were considered as "good knowledge" otherwise considered as "poor knowledge" regarding malaria prevention. Behavioral or practice of participants regarding to malaria prevention and control strategies was assessed using a 7-item questionnaire where report of at least one prevention and control strategy related to malaria is considered as having "good behavior or practice".

\subsection{Data Quality Control}

Pre-testing was done on 8 respondents $(5 \%$ of the sample size) in Bonke district who were chosen to ensure that the questions are easily understood and all the data collectors were understood each question in a similar way and collect the intended information. The result of the pre-test was used to improve the phrasing and modification of questions in the questionnaire. The supervisors checked the questionnaires for completeness every day. Incomplete questionnaires were returned to data collectors for correction by revisiting the households.

\subsection{Data Analysis}

Data were coded and entered in to SPSS-for windows version 20 for analysis. Frequency distribution tables were used to quantify socio-demographic variables, knowledge and attitude of respondents related to symptoms, causes, transmission, prevention and control measures of malaria, and practices toward malaria prevention and control methods.

Bivariate logistic regression analysis was done to determine the presence and degree of association between KAP and socio-demographic variables. Odds Ratios (OR) with $95 \%$ confidence intervals was calculated. Variables having p-value less than 0.05 were treated as showed a statistically significant association with KAP.

\subsection{Ethical Consideration}

The study obtained ethical clearance from the Institutional Review Board of Arba Minch University. Supportive letter was obtained from the Bonke district health office before data collection. Before interviewing the respondents, all the participants were given an explanation about the aim of the study, and verbal informed consent was secured. In addition they were told that they had the right to terminate or refuse to participate in the study.

\section{Results}

\subsection{Socio-demographic Characteristics}

A total of 159 individual were interviewed in this study, most of the respondents were females $89(55.9 \%)$, aged $26-35$ years $48(30.2 \%)$, illiterate $74(46.5 \%)$, Orthodox by religion $66(41.5 \%)$ and married $107(67.2 \%)$. In this study, most of the interviewees were found to be housewives and farmers, their respective coverage was 55(34.6\%) and the number of people involved from the government employee were only $15(9.3 \%)$ (Table 1).

Table 1. Socio-demographic characteristics of the study participants, Dembele Kebele, Southern Ethiopia, 2017.

\begin{tabular}{llll}
\hline Variables & Category & Frequency & $\begin{array}{l}\text { Percentage } \\
(\%)\end{array}$ \\
\hline \multirow{2}{*}{ Sex } & Male & 70 & 44.1 \\
& Female & 89 & 55.9 \\
& $15-25$ & 44 & 27.7 \\
Age & $26-35$ & 48 & 30.2 \\
& $36-45$ & 41 & 25.8 \\
& $>45$ & 26 & 16.3 \\
\hline
\end{tabular}




\begin{tabular}{llll}
\hline Variables & Category & Frequency & $\begin{array}{l}\text { Percentage } \\
(\%)\end{array}$ \\
\hline \multirow{4}{*}{ Education } & Illiterate & 74 & 46.5 \\
& Elementary and Junior & 55 & 34.6 \\
& Secondary and above & 30 & 18.9 \\
& Orthodox & 66 & 41.5 \\
Religion & Protestant & 74 & 46.6 \\
& Catholic & 15 & 9.4 \\
& Muslim & 4 & 2.5 \\
& Single & 33 & 20.8 \\
& Married & 107 & 67.2 \\
& Divorced & 15 & 9.5 \\
& Widowed & 4 & 2.5 \\
& Government employee & 15 & 9.4 \\
& Housewife & 55 & 34.6 \\
& Merchant & 26 & 16.3 \\
& Farmer & 55 & 34.6 \\
& Others & 8 & 5.1 \\
\hline
\end{tabular}

\subsection{Knowledge and Attitude Toward Malaria Transmission and Preventive Methods}

Amharic version of Malaria is known as "webba", which is most commonly used term in Ethiopia. The conducted interview survey results showed that all respondents had ever heard about malaria and among them more than $86 \%$ of them believed that malaria was one of the most health problem diseases. Most of the respondents $133(83.7 \%)$ believed that malaria can be transmitted through mosquito bite and 126 (79.3\%) understood that malaria cannot transmit through flies. Almost $132(83.0 \%)$ and $141(88.7 \%)$ of the participant were thought that malaria could not be transmitted from one person to another via respiratory route and bodily contact with patients respectively. One hundred and thirty six $(85.5 \%)$ of the participants were replied that malaria is a preventable disease. In the present study, nearly $136(85.5 \%)$ of the respondents were mentioned mosquito nets as best malaria preventive methods and $128(80.5 \%)$ of participants were understood that mosquito nets protect from mosquito bite (Table 2).

Table 2. Knowledge and attitude of respondents regarding transmission and preventive methods of malaria, Dembele Kebele, Southern Ethiopia, 2017.

\begin{tabular}{lll}
\hline Variables & Frequency & $\begin{array}{l}\text { Percentage } \\
(\%)\end{array}$ \\
\hline $\begin{array}{l}\text { Have hear about malaria? } \\
\text { Yes }\end{array}$ & 159 & 100 \\
No & 0 & 0 \\
Do you think malaria is one of the most & & \\
health problem diseases of the community? & 136 & 86.7 \\
Yes & 23 & 13.3 \\
No & & \\
Mode of transmission & 133 & 83.7 \\
Malaria transmits through mosquito bite & 21 & 13.2 \\
Yes & 5 & 3.1 \\
No & & \\
Don't know & & \\
Malaria transmits from one to other via & 11 & 6.9 \\
respiratory route & 132 & 83.0 \\
Yes & & \\
No & & \\
\hline
\end{tabular}

\begin{tabular}{lll}
\hline Variables & Frequency & $\begin{array}{l}\text { Percentage } \\
\text { (\%) }\end{array}$ \\
\hline Don't know & 16 & 10.1 \\
Malaria transmits through bodily contact & & \\
with patients & 7 & 4.4 \\
Yes & 141 & 88.7 \\
No & 11 & 6.9 \\
Don't know & & \\
Malaria transmits by flies & 26 & 16.3 \\
Yes & 126 & 79.3 \\
No & 7 & 4.4 \\
Don't know & & \\
Preventive methods & & \\
Malaria can be prevented & 136 & 85.5 \\
Yes & 23 & 14.5 \\
No & & \\
Personal precautions measures* & 96 & 60.4 \\
Take tablets & 136 & 85.5 \\
Use of mosquito net & 128 & 80.5 \\
House spray (DDT) & 130 & 81.7 \\
Drain stagnant water & 118 & 74.2 \\
Closing windows and doors & 121 & 76.1 \\
Fumigation & 75 & 47.2 \\
Clear the vegetation & 128 & 80.5 \\
Advantage of mosquito nets* & 117 & 73.5 \\
Protect from mosquito bite & 5 & 13.8 \\
Avoid getting malaria & & 8.8 \\
Protect from other insects & & 3.1 \\
Sleep better & & \\
Warmer & & \\
\hline & & \\
\hline
\end{tabular}

* More than one answer is possible

\subsection{Knowledge and Attitude toward Cause of Malaria, Mosquito Breeding Site and Biting Time}

Table 3 shows the respondents' knowledge and attitudes towards cause of malaria, mosquito breeding site and biting time. Almost $98(61.7 \%)$ and $54(34.0 \%)$ of the participants were believed that mosquitoes are bite humans at night during sleeping time and breed in Stagnant water and swampy area respectively. More than half of the respondent were said that, dry season is seasonal cause of malaria in the study area. One hundred and twelve (70.4\%) of the respondents reported the cause of malaria is being bitten by mosquitoes. Some of respondents also replied that lack of personal hygiene $21(13.2 \%)$, chewing maize stalk $17(10.7 \%)$ and hunger $9(5.7 \%)$ as causes of malaria (Table 3$)$.

Table 3. Knowledge and attitude of respondents related to cause of malaria, mosquito breeding site and biting time, Dembele Kebele, Southern Ethiopia, 2017.

\begin{tabular}{lll}
\cline { 1 - 2 } Variables & Frequency & Percentage (\%) \\
\cline { 1 - 2 } Mosquito breeding site & 51 & 32.1 \\
Stagnant water and swampy area & 54 & 34.0 \\
Running water & 20 & 12.6 \\
Houses & 15 & 9.4 \\
Vegetation & 12 & 7.5 \\
Don't know & 7 & 4.4 \\
Cause of malaria mentioned & & \\
Mosquito bite & 112 & 70.4 \\
Lack of personal hygiene & 21 & 13.2 \\
Chewing maize stalk & 17 & 10.7 \\
Hunger & 9 & 5.7 \\
\hline
\end{tabular}




\begin{tabular}{lll}
\hline Variables & Frequency & Percentage (\%) \\
\hline Mosquito breeding site & & \\
\hline Seasonal cause of malaria & 49 & 30.8 \\
Cloudy weather & 44 & 27.7 \\
Rainy season & 52 & 32.7 \\
Dry season & 14 & 8.8 \\
Any season & & \\
When mosquitoes bite human mostly & 98 & 61.7 \\
Night & 11 & 6.9 \\
Day & 42 & 26.4 \\
Any time & 8 & 5.0 \\
Not sure & & \\
\hline
\end{tabular}

\subsection{Housing Condition, Distance of House from Breeding} Site of Mosquito and DDT Spray Case

About 126(79\%), 112(70.4\%) and 112(70.4\%) of the respondents house had windows (holes), shared with animal and less than or equal to $400 \mathrm{~m}$ distance from the breeding site respectively. Out of the total respondents, 118 (74.2\%) reported that their houses were sprayed with DDT in the last 12 months and about $41(25.8 \%)$ mentioned that their houses were not sprayed within the last 12 months (Table 4).

Table 4. Housing condition, distance of house from breeding site of mosquito, and DDT spray case, Dembele Kebele, Southern Ethiopia, 2017.

\begin{tabular}{lll}
\hline Variables & Frequency & Percentage (\%) \\
\hline Housing condition & & \\
Hes window & 126 & 79.0 \\
No & 33 & 21.0 \\
Shared with animal & & \\
Yes & 112 & 70.4 \\
No & 47 & 29.6 \\
Distance of breeding site & & \\
$\leq 400 \mathrm{~m}$ & 112 & 70.4 \\
$>400 \mathrm{~m}$ & 47 & 29.6 \\
DDT spray & & \\
Yes & 118 & 74.2 \\
No & 41 & 25.8 \\
\hline
\end{tabular}

\subsection{Practices of Respondents Towards Malaria Prevention and Control Strategies}

The use of mosquito net $121(76.1 \%)$ and draining stagnant water $98(61.6 \%)$ are the two most frequently applied methods for malaria prevention by the respondents. About $44.7 \%$ and $43.4 \%$ respondents were practiced house spray with insecticide and closing windows and doors as malaria preventive methods and mosquitoes control strategy respectively (Table 5).

Table 5. Practices of respondents towards malaria prevention and control, Dembele Kebele, Southern Ethiopia, 2017.

\begin{tabular}{|c|c|c|}
\hline \multirow{2}{*}{$\begin{array}{l}\text { Variables } \\
\text { Practices toward malaria prevention and } \\
\text { Control mentioned* }\end{array}$} & \multirow[b]{2}{*}{ Frequency } & \multirow{2}{*}{$\begin{array}{l}\text { Percentage } \\
(\%)\end{array}$} \\
\hline & & \\
\hline Use of mosquito net & 121 & 76.1 \\
\hline Drain stagnant water & 98 & 61.6 \\
\hline House spray with insecticide & 71 & 44.7 \\
\hline Closing windows and doors & 69 & 43.4 \\
\hline Fumigation & 51 & 32.1 \\
\hline Take tablets & 33 & 20.8 \\
\hline Clear the vegetation & 28 & 17.6 \\
\hline Don't use & 22 & 13.8 \\
\hline
\end{tabular}

* More than one answer is possible

\subsection{Knowledge of Signs and Symptoms of Malaria}

A total of $153(96.2 \%)$ respondents knew at least one correct sign/symptom of malaria. Thirteen percent respondents gave wrong responses while 6(3.8\%) said they didn't know any sign or gave no response. The most popular sign of malaria was abdomen pain followed by fever, headache, chill, muscles pain, vomiting, thirsty and loss of appetite. Wrong sign/symptom included yellow urine (Figure 1).

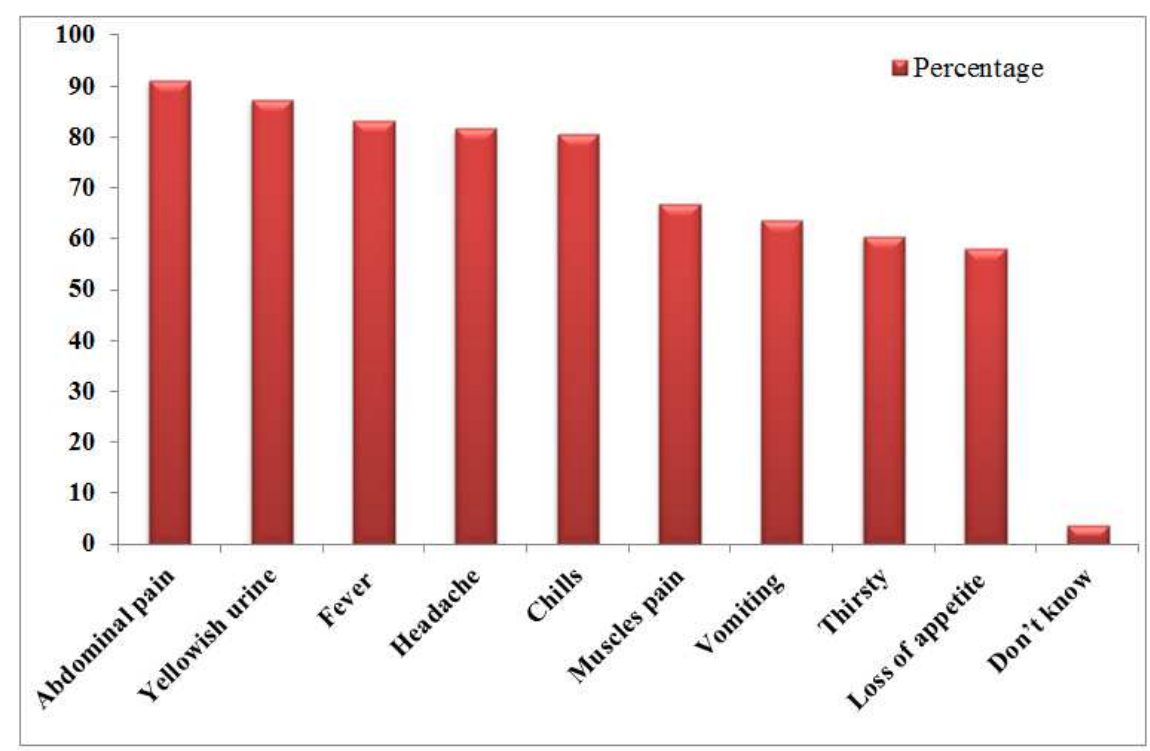

Figure 1. Knowledge of respondents about the symptoms of malaria, Dembele Kebele, Southern Ethiopia, 2017. 


\subsection{Factors Associated with Knowledge of Malaria Transmission and Prevention Methods}

One hundred eighty $(80.5 \%)$ of participants were having good knowledge regarding malaria transmission whereas $118(74.2 \%)$ of participants were having good knowledge regarding to malaria prevention.

By applying binary logistic regression analysis, the result showed that knowledge on mode of malaria transmission was positively associated with sex (male) $(\mathrm{OR}=3.3$; CI, 1.277.22), age $(\mathrm{OR}=5.03$; $\mathrm{CI}, 1.03-24.46)$ and education level $(\mathrm{OR}=3.30 ; 95 \% \mathrm{CI}, 1.04-10.50)$. Among socio-demographic variables, only education level $(\mathrm{OR}=3.8 ; \mathrm{CI}, 1.51-9.65)$ was significance factor to knowledge on malaria prevention. While other socio-demographic variables were doesn't showed significantly associated with knowledge on malaria transmission and prevention (Table 6).

Table 6. Bivariate analysis between socio-demographic variables and knowledge on malaria transmission and prevention, Dembele Kebele, Southern Ethiopia, 2017.

\begin{tabular}{|c|c|c|c|c|c|c|c|c|}
\hline \multirow{2}{*}{ Explanatory Variables } & \multicolumn{4}{|c|}{ Knowledge on transmission } & \multicolumn{4}{|c|}{ Knowledge on prevention } \\
\hline & Good & Poor & OR $(95 \%$ CI $)$ & P-value & Good & Poor & OR $(95 \%$ CI $)$ & P-value \\
\hline \multicolumn{9}{|l|}{ Sex } \\
\hline Male & 62 & 8 & $3.27(1.27-7.22)$ & 0.01 & 53 & 17 & $1.15(0.56-2.36)$ & 0.7 \\
\hline \multicolumn{9}{|l|}{ Age } \\
\hline $15-25$ & 31 & 13 & 1 & & 29 & 15 & 1 & \\
\hline $26-35$ & 37 & 11 & $1.4(0.55-3.58)$ & 0.46 & 35 & 13 & $1.39(0.57-3.39)$ & 0.46 \\
\hline $36-45$ & 34 & 7 & $2.03(0.72-5.76)$ & 0.17 & 32 & 9 & $1.8(0.69-4.83)$ & 0.12 \\
\hline$>45$ & 24 & 2 & $5.03(1.03-24.46)$ & 0.03 & 22 & 4 & $2.8(0.82-9.77)$ & 0.08 \\
\hline \multicolumn{9}{|l|}{ Education } \\
\hline Illiterate & 49 & 25 & 1 & & 46 & 28 & 1 & \\
\hline Elementary and junior & 51 & 4 & $0.50(0.11-2.20)$ & 0.36 & 44 & 7 & $3.8(1.51-9.65)$ & 0.00 \\
\hline Secondary and above & 26 & 4 & $3.30(1.04-10.50)$ & 0.03 & 28 & 2 & $8.52(1.88-38.5)$ & 0.00 \\
\hline
\end{tabular}

\section{Discussion}

One of the main concerns of this study was to assess knowledge and awareness of communities toward the cause, transmission, prevention and control measures of malaria and its association with mosquitoes using community-based cross-sectional study in Dembele kebele, southern Ethiopia.

The conducted interview survey results indicated that all respondents had ever heard of malaria and more than $86 \%$ of them believed that malaria was one of the most health problem diseases of the society affecting both sex and all age groups, which is consistent with previous reports [29]. More than $80 \%$ of participants responded that mosquito bites as the mode of malaria transmission and the transmission occurs from person-to-person through mosquito bite. This awareness is higher than that the study conducted in India (72.3\%) and Tanzania (range 59-79\%) [5, 22], but lower the level reported in Shewa Robit town, Ethiopia [21]. This might be due to low coverage of Information Communication and Education (ICE), which is the community haven't sufficient information about malaria from health extension workers in the current study area.

In spite of the fact that majority of the respondents associated the cause of malaria with mosquito bite, more than $16 \%$ of participants mentioned flies, chewing maize stalk, via respiratory route, hunger and body contact with malaria patient as the causes of malaria. These types of misunderstandings were previously mentioned by other studies $[21,25]$.

However, some of the respondents in the present study were thought that chewing maize stalk and hunger as direct causes of malaria due to misconception about risk factors of malaria. Bear in mind, such kind of idea may result from the occurrence of other related factors with stated condition. For instance, following rainy season maize pollen might be released, which can be used as food source for larval stage enhancing their development and survival. Thus, the rate of malaria transmission could be increased in cold weather during winter seasons at the same time maize become common [30]. In addition, poor nutritional status due to hunger could also make individuals more susceptible to malaria [31]. These misunderstanding of the respondents on the direct cause of malaria may influence the actual prevention mechanism they may choose. Thus, this knowledge gap should be filled with appropriate health education. Health education brought better understanding and awareness of the causes of malaria in the communities were reported in the previous studies [32].

Hygiene and sanitation is an important component of the malaria control programme and it is a message of "maintaining environmental sanitation" [33]. They input this sensitization particular part is frequently used in malaria prevention and control interventions. However, in this study only $13.2 \%$ of respondents were mentioned that lack of personal hygiene and sanitation is cause of malaria; this awareness is very lower than the level reported in Central African Republic [25].

In the present study, more than half of the study participants responded that mosquitoes' habit of biting during night/sleeping time. This finding is higher than the study conducted in rural part of central Ethiopia (42.6\%) [34]. Therefore, this level of perception among respondents in this study is encouraging to take appropriate preventive measures and proper use of mosquito nets. However, this finding was lower than the study conducted in Shewa (83.8\%) [21]. Among mosquito breeding sites, the participants responded 
that stagnant water and swampy area was $34 \%$, which was lower than the finding of central Ethiopia (36.2\%) [34] and Shewa $(91.6 \%)$ [21], the indication of the highest result in Shewa might be due to the used of multiple responses for each category.

The majority of participants were responded that cloudy weather is the seasonal cause of malaria. The result of the present study is lower than the study conducted in the communities of four districts of the Central African Republic (range 48-79.7\%) [25]. Some respondents still believed that exposure to cold/cloudy weather is a direct cause of malaria disease. This belief of the participants might have stemmed from the concurrence of cold and cloudy weather could be related to the presence of mosquito breeding sites.

In this study, more than $85 \%$ of respondents believed that malaria is preventable disease which is higher than the finding of a study in south-western Saudi Arabia (30.2\%) [35]. The main types of malaria preventive measures frequently reported by the present study participants were use of mosquito net, drain stagnated water (mosquito breeding sites), house spray (DDT) and fumigation. This finding was consistence with previous studies from other part of Ethiopia, Tanzania, India, and Saudi Arabia [21, 35, 36, 37]. In this study, $(85.5 \%)$ respondents knew that mosquito nets prevent malaria, and hence made use of them to prevent the disease. However, among them only (76.1\%) of participants used ITNs. Therefore, this gap indicates that study participants having the problem of practices toward malaria prevention and control strategies throughout INTs.

The relatively high rate $(>50 \%)$ of our study respondents who were well-known with the sign and symptoms of malaria is a cause for concern. Similar studies conducted in Ethiopia indicate that respondents were mentioned fever, chills and headache are as a symptom of malaria [26]. Other studies reported that respondents had poor knowledge about malaria signs and symptoms and it may delay treatment [25].

Among socio-demographic variables, sex, age and education were found to be significantly associated with knowledge on transmission of malaria whereas only education status was significant factor to knowledge on prevention of malaria. Male participants were more than 3 times more likely to be knowledgeable on transmission of malaria than the female participants. This finding was similar with the study conducted in India [37]. In the present study, among participants, age group greater than 45 were more than 5 times more likely to be knowledgeable on transmission of malaria than the respondents of age group less than 25 , however the present finding is contradicted with the study conducted in district of Orissa [37].

In addition, having education level of secondary and above were more than 3 times more likely to be knowledgeable on transmission of malaria than illiterate participants. This result was consistent with the study conducted in Cameroon and India [23, 37]. Whereas having education level of above secondary and elementary and junior were more than 8 and 3 times more likely to be knowledgeable on prevention method of malaria than illiterate participants respectively, similarly this variable was significant factor in other the study [37].

In fact, education in school is the primary source of information regarding about malaria transmission and prevention since it has been explained lessons on malaria in school, even the level of education is strongly associated with the level of their understanding. Therefore, education clearly influences knowledge about modes of malaria transmission. Educated communities also have multiple sources of information when compared with their uneducated counterparts [38].

\section{Conclusion}

This study aimed to assess the overall communities' knowledge and awareness regarding the cause, transmission, and preventive measures of malaria; it was found to be high in Dembele kebele. The current community awareness on symptoms of malaria and use of mosquito net as protective measure against mosquito bite is also fair. However, there are still knowledge gap on direct cause of malaria, therefore, appropriate health education should be implemented to come across this gap. Moreover, attention should be focused on the residents near to breeding sites and people who shared their house with animals. This may contribute to higher prevalent rate than those who live far from breeding site and made another house for their animals. Strengthening community based organizations is the most important factor to improve malaria prevention and control strategy in the study area.

\section{Acknowledgements}

The authors are very grateful to heads of Bonke district health office, for its administrative and technical assistance. Interviewers and supervisors deserves especial acknowledgement for data collection and care full supervision respectively, and also thankful to Dembele kebele communities who participated in this study. Last but not least, the authors would like to thank Hub Jema'a members especially Jemil Abdella and Mensur Abdella for their transportation facilities.

\section{References}

[1] Joel G B, Martin S A and Anne M (2004). Conquering the Intolerable burden of Malaria: What's New, What's needed: A Summary. The American Journal of Tropical Medicine and Hygiene, 71(2):1-15.

[2] Seyoum A, Balcha F and Balkew M (2002). Impact of cattle keeping on human biting rate of Anopheline Mosquitoes and malaria transmission around Ziway, Ethiopia. East African Medical Journal, 9(9):485-490.

[3] FMOH, Malaria diagnosis and treatment guidelines for health workers in Ethiopia, 2nd edition, Addis Ababa, July 2004; PP 1-2.

[4] Ethiopian Public Health Association (EPHA) (2004). Proceeding of the Panel Discussion on The 2003 Malaria Epidemics in Ethiopia; Addis Ababa, 6-28. 
[5] Mathania M, Kimera S I and Silayo R S (2016). Knowledge and awareness of malaria and mosquito biting behaviour in selected sites within Morogoro and Dodoma regions Tanzania. Malaria Journal, 15(287):1-9.

[6] Mitiku K, Mengistu G and Gelaw B (2003). The reliability of blood film examination for malaria at the peripheral health unit. Ethiopia Journal of Health Development, 17(3):197-204.

[7] Castro M C, Yamagata Y and Deomtaslwa (2004). Integrated urban malaria control: A case study in Dares Salaam, Tanzania. The American Journal of Tropical Medicine and Hygiene, 71(2):103-117.

[8] Kate M, Joseph K and Stephen S (2002). Examining the determinants of mosquito-avoidance practice in two Kenyan cities. Malaria Journal, 1:14, DOI: 10.1186/1475-2875-1-14 Available at http://www.malariajournal.com/content/1/1/14.

[9] Ghebreyesus T A, Witten K H, Getachew A, Yohannes A M, Tesfay W, Minass M, Bosman A, and Teklehaimanot A (2000). The community Based Malaria Control Program in Tigray, Northern, Ethiopia. The Review of Program Set-up, Activities, Outcomes and Impact. Parassitologia, 42(34):255-290.

[10] Regmi K, Kunwar A and Ortega L (2016). A systematic review of knowledge, attitudes and beliefs about malaria among the South Asian population. Infection Ecology and Epidemiology, 6:30822.

[11] [11] Richard C, Kamini N M and Donald R (2000). Spatial targeting of interventions against malaria. Bulletin of the World Health Organization, 78:1401-1411.

[12] Abose T, Yeebiyo Y and Olana D (1998). Re-orientation and definition of the role of malaria vector control in Ethiopia. WHO, Division of Control of Tropical Diseases. WHO/MA/98.1085; PP 1-9.

[13] Sarah G, Staedke E, Willis N, Cox J, Moses R. Kamya, Philip J, Rosenthal and Dorsey G (2003). Short Report: Proximity to Mosquito Breeding Sites as a Risk factor for Clinical Malaria Episodes in an Urban cohort of Uganda Children. The American Journal of Tropical Medicine and Hygiene, 69(3):244-246.

[14] Robert V, Macintrye K and Keating J(2003). Malaria transmission in urban sub-Saharan Africa. The American Journal of Tropical Medicine and Hygiene, 68(2):169-176.

[15] Keiser J, Utzinger J, Castro M C, Smith T A, Tanner M and Singer B H (2004). Urbanization in sub-Saharan Africa and Implication for Malaria Control. The American Journal of Tropical Medicine and Hygiene, 71(2):118-127.

[16] Fantahun M and Degu G (2004). Burden of Diseases in Amhara Region, Ethiopia. Ethiopia Medicinal Journal, 42(3):165-172.

[17] Fantahun M and Degu G (2003). Health Service Utilization in Amhara Region of Ethiopia. Ethiopia Journal of Health Development, 17(2):141-147.

[18] Planning and Programming Department, FMOH, Health and health related indicators, Feb. 2004; PP 38-43.

[19] [Knudsen A B and Slooff R (1992). Vector-borne disease problems in rapid urbanization: new approaches to vector control. Bulletin of the World Health Organization, 70(1):1-6.
[20] The Federal Democratic Republic of Ethiopia, Ministry of Health, Malaria Control Profile: Addis Ababa, March 2000; PP 1-10.

[21] Abate A, Degarege A and Erko B (2013). Community knowledge, attitude and practice about malaria in a low endemic setting of Shewa Robit Town, northeastern Ethiopia. BMC Public Health, 13(312):1-8.

[22] Das A, Gupta D R K, Friedman J, Pradhan M M, Mohapatra C C and Sandhibigraha D (2013). Community perceptions on malaria and care-seeking practices in endemic Indian settings: policy implications for the malaria control programme. Malaria Journal 12(39):1-12.

[23] Kimbi K H, Nkesa S B, Ndamukong-Nyanga J L, Sumbele I U N, Atashili J and Atanga M B S (2014). Knowledge and perceptions towardsmalaria prevention among vulnerable groups in the Buea Health District, Cameroon. BMC Public Health 14(883).

[24] Fuge T G, Ayanto S Y and Gurmamo F L (2015). Assessment of knowledge, attitude and practice about malaria and ITNs utilization among pregnant women in Shashogo District, Southern Ethiopia. Malaria Journal, 14(235):1-9.

[25] Serengbe G B, Moyen J M, Fioboy R, Beyam E N, Kango C, Bangue C and Manirakiza A (2015). Knowledge and perceptions about malaria in communities in four districts of the Central African Republic. BMC Research Notes, 8(162):1-6.

[26] Ayalew A (2010). Knowledge And Practice Of Malaria Prevention Methods Among Residents Of Arba Minch Town And Arba Minch Zuria District, Southern Ethiopia. Ethiopian Journal of Health Science, 20(3):185-193.

[27] Sood R D, Mittal P K, Kapoora N, Razdan R K, Dua V K and Dash A P (2010). Community awareness, perceptions, acceptability and preferences for using LLIN against malaria in villages of Uttar Pradesh, India. Journal of Vector Borne Disease, 47:243-248.

[28] Bonke woreda/Town administration health office: Bonke woreda/Town administration health office 2016/2017 report. Bonke woreda/Town, Southern region: Bonke woreda/Town health center; 2017.

[29] Lora L S, Abanish R, Mohamed I B, Mrigendra P S, Jordan T, Blair J W, Katherine M J, Kojo Y A, Neeru S and Davidson H $\mathrm{H}$ (2010). Attitudes, knowledge, and practices regarding malaria prevention and treatment among pregnant women in eastern India. The American Journal of Tropical Medicine and Hygiene, 82(supp 6):1010-1016.

[30] Kebede A, Mccann J C, Kiszewski A E and Ye-ebiyo Y (2005). New evidence of the effects of agro-ecologic change on malaria transmission. The American Journal of Tropical Medicine and Hygiene, 4(supp 73):676-680.

[31] Ayele D G, Zewotir T T and Mwambi H G (2012). Prevalence and risk factors of malaria in Ethiopia. Malaria Journal, 11:195.

[32] Dickson S N, Anna L N, Henri L F K, Jules C N A, Charles S W, Sarah M N and Alfred K N (2011). Knowledge and practices relating to malaria in Ndu community of Cameroon: Signs and symptoms, causes and prevention. Journal of Public Health and Epidemiology, 3(supp 6):294-300.

[33] Ghosh S K, Patil R R, Tiwari S and Dash A P (2006). A community-based health education programme for bioenvironmental control of malaria through folk theatre (Kalajatha) in rural India. Malaria Journal, 5:123. 
[34] Legesse M and Deressa W (2009). Community awareness about malaria, its treatment and mosquito vector in rural highlands of central Ethiopia. Ethiopian Journal of Health Development, 23(supp 1):40-47.

[35] Khairya S, Al-Surimi K, Ali A, Shubilyd H M, Al Walaane N, Househ M, and El-Metwally A (2017). Knowledge, attitude and practice about malaria in south-western Saudi Arabia: A household-based cross-sectional survey. Journal of Infection and Public Health, available at http://www.elsevier.com/lo cate/jiph.

[36] Safari M K, Fabian M, Joseph R M, Soori E N, Godfrey M K, Robert M, Coleman K, Stephen M and Leonard E G M (2010). Knowledge, attitudes and practices about malaria among communities: Comparing epidemic and non-epidemic prone communities of Muleba district, North-western Tanzania. BMC Public Health, 10:395.

[37] Das A and Ravindran T K S (2011). Community knowledge on malaria among febrile patients in an endemic district of Orissa, India. Journal of Vector Borne Disease, 48:46-51.

[38] Adedotun A A, Morenikeji O A and Odaibo A B (2010). Knowledge, attitudes and practices about malaria in an urban community in South-Western Nigeria. Journal of Vector Borne Disease, 47:155-159. 